\title{
Title: Catalytic enantioselective Minisci-type addition to heteroarenes
}

\author{
Authors: Rupert S. J. Proctor, Holly J. Davis and Robert J. Phipps*.
}

\author{
Affiliation: \\ Department of Chemistry, University of Cambridge, Lensfield Road, Cambridge, CB2 1EW, \\ United Kingdom. \\ *Correspondence to: rjp71@cam.ac.uk
}

One Sentence Summary: A chiral Brønsted acid and an iridium photocatalyst enable catalytic enantioselective Minisci-type addition of prochiral radicals to pyridines and quinolines.

\begin{abstract}
Basic heteroarenes are a ubiquitous feature of pharmaceuticals and bioactive molecules, and Minisci-type additions of radical nucleophiles are a leading method for their elaboration. Despite many Minisci-type protocols that result in the formation of stereocenters, exerting control over the absolute stereochemistry at these centers remains an unmet challenge. We report a process for addition of prochiral radicals, generated from amino acid derivatives, to pyridines and quinolines with excellent control of both enantioselectivity and regioselectivity. An enantiopure chiral Brønsted acid catalyst serves both to activate the substrate and induce asymmetry, while an iridium photocatalyst mediates the required electron transfer processes. We anticipate that this method will expedite access to enantioenriched small-molecule building blocks bearing versatile basic heterocycles.
\end{abstract}

\section{Main Text:}

Heteroarenes with basic nitrogen centers, of which pyridines and quinolines are the most common classes, are ubiquitous in pharmaceuticals, agrochemicals and small molecules of medicinal interest $(1,2)$. The nitrogen's basicity precludes traditional electrophilic aromatic substitution pathways, necessitating alternative strategies for elaboration. One widely used protocol is the addition of free radicals to protonated heterocycles, pioneered by Minisci in the 1960s and 1970s and often referred to as 'Minisci-type' chemistry (3-6). Recent renewed interest has led to numerous advances in radical generation, allowing for milder conditions and convenient radical precursors (7-11). Furthermore, photoredox catalysis (12-14) has provided exciting avenues for radical generation in Minisci-type additions with precursors that include alcohols $(15,16)$, ethers (17), boronic acids (18), carboxylic acids (19), and redox-active esters $(20,21)$. However, control over absolute stereochemistry in the addition of prochiral radicals has proven elusive, despite many of the aforementioned protocols resulting in the formation of a stereocenter at the benzylic position. 
A Typical approaches to enantioenriched $\alpha$-heterocyclic amine derivatives:

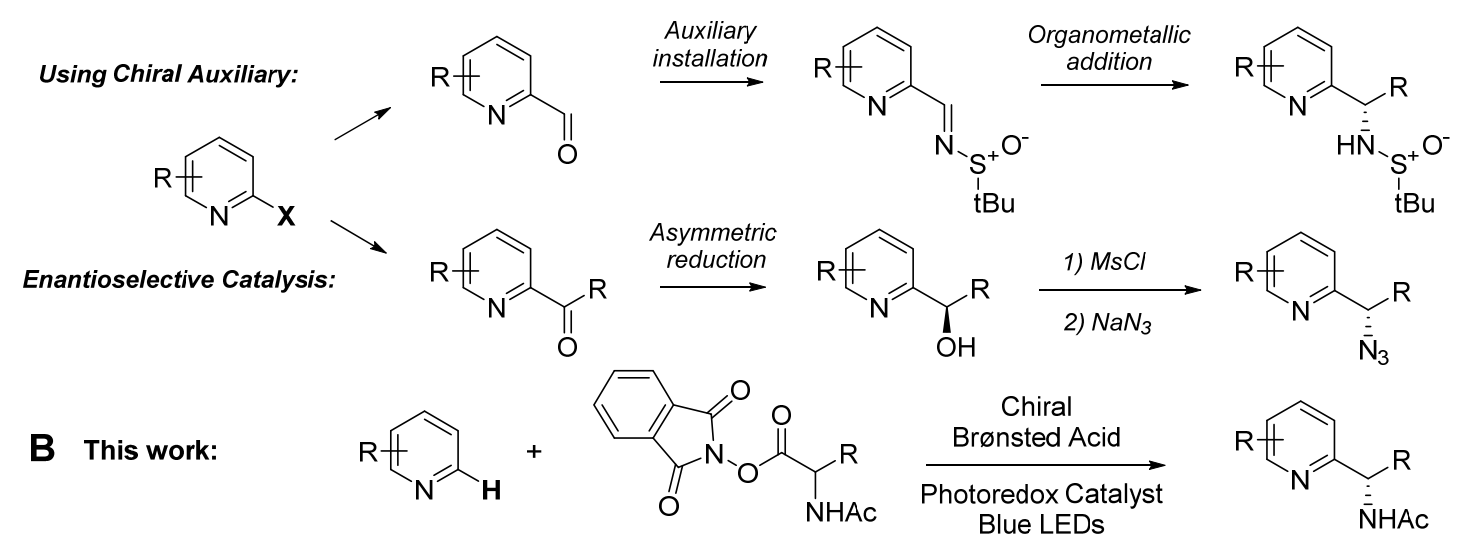

Fig. 1. Strategies to access enantioenriched $\alpha$-heterocyclic amines. (A) Typical current approaches. (B) The method reported herein. Ms, methanesulfonyl; Ac, acetyl.

There is an increasing drive in the pharmaceutical industry for higher degrees of threedimensionality in lead compounds, which necessitates asymmetric introduction of chiral centers (22). Realization of a catalytic enantioselective Minisci-type addition would constitute a powerful and efficient strategy for the enantioselective synthesis of basic heteroarenes bearing adjacent stereocenters. To put this prospect into context, a typical existing approach to enantioenriched $\alpha$-pyridyl amines involves diastereoselective addition of organometallics to enantiopure pyridyl imines derived from Ellman's sulfonamide auxiliary (Fig. 1A, upper scheme) (23). Stereospecific coupling of pyrrolidine-derived chiral boronic esters has also been demonstrated (24). Approaches using asymmetric catalysis are typically lengthy, involving asymmetric reduction of a pyridyl ketone, conversion to a leaving group and azide displacement (lower scheme). An alternative catalytic route could involve installation of an enamide functionality onto the pyridine ring, followed by asymmetric hydrogenation (25) and a recent strategy merged nickel catalysis and photoredox catalysis to access 3- and 4-substituted pyridines (26). In all cases, a functional group must first be installed on the pyridine scaffold in a regioselective manner, necessitating additional chemical steps. Here we disclose the direct addition of $\alpha$-amino alkyl radicals to the 2-position of basic heteroarenes by virtue of a combination of asymmetric Brønsted acid catalysis and photoredox catalysis (Fig 1B). This catalytic approach does not require prefunctionalization of the heterocycle and allows excellent control of both enantioselectivity and regioselectivity. The products generated possess structural features highly desirable in pharmaceutical compounds: a basic heteroarene, protected primary amine and a defined stereocenter, all in close proximity. In addition, this work constitutes a rare case of non-covalent organocatalysis being applied to control enantioselectivity in a single electron process (27-33).

In considering a strategy towards this goal, we surmised that Minisci chemistry should, in principle, be amenable to asymmetric Brønsted acid catalysis (34). The heteroarene LUMO is significantly lowered upon protonation and the conjugate anion of the chiral acid should remain associated with the pyridinium cation through electrostatic and hydrogen bonding interactions, providing substrate activation in a chiral environment. Encouragingly, Shang, Fu and co-workers recently reported that catalytic amounts of achiral or racemic Brønsted and Lewis acids can be used to promote Minisci additions, in place of the stoichiometric acid that is typically employed 
(20, 21, 35, 36). Upon careful consideration of the proposed mechanism of Minisci-type additions (3), we envisaged two plausible scenarios that may lead to enantioenrichment in the product using a chiral Brønsted acid. First, a bifunctional conjugate anion, such as a chiral phosphate, might engage in hydrogen bonding interactions with a suitable radical nucleophile as well as its pyridinium counterion (Fig 2A, I). In this manner, a well-defined ternary transition state for enantiodetermining radical addition may occur. Following radical addition, the next step would be deprotonation of the aminium ion intermediate $\boldsymbol{I I}$ before typically fast single electron oxidation of the resulting neutral radical III. Inspiring studies from Minisci and co-workers suggested that the addition of stabilized nucleophilic radicals to protonated heteroarenes can be reversible and that in such cases the irreversible, product-determining step of the process is the subsequent deprotonation (37). This introduces the second scenario: that deprotonation of II could be product-determining and either carried out by the associated chiral phosphate or an external base in the chiral environment that the phosphate provides. In this case, reversible radical addition may result in a situation where both the relative stabilities of various diastereomers of $\boldsymbol{I I}$ and their rates of deprotonation could play important roles in determining product enantioselectivity. 
A Mechanistic considerations for asymmetric radical addition:

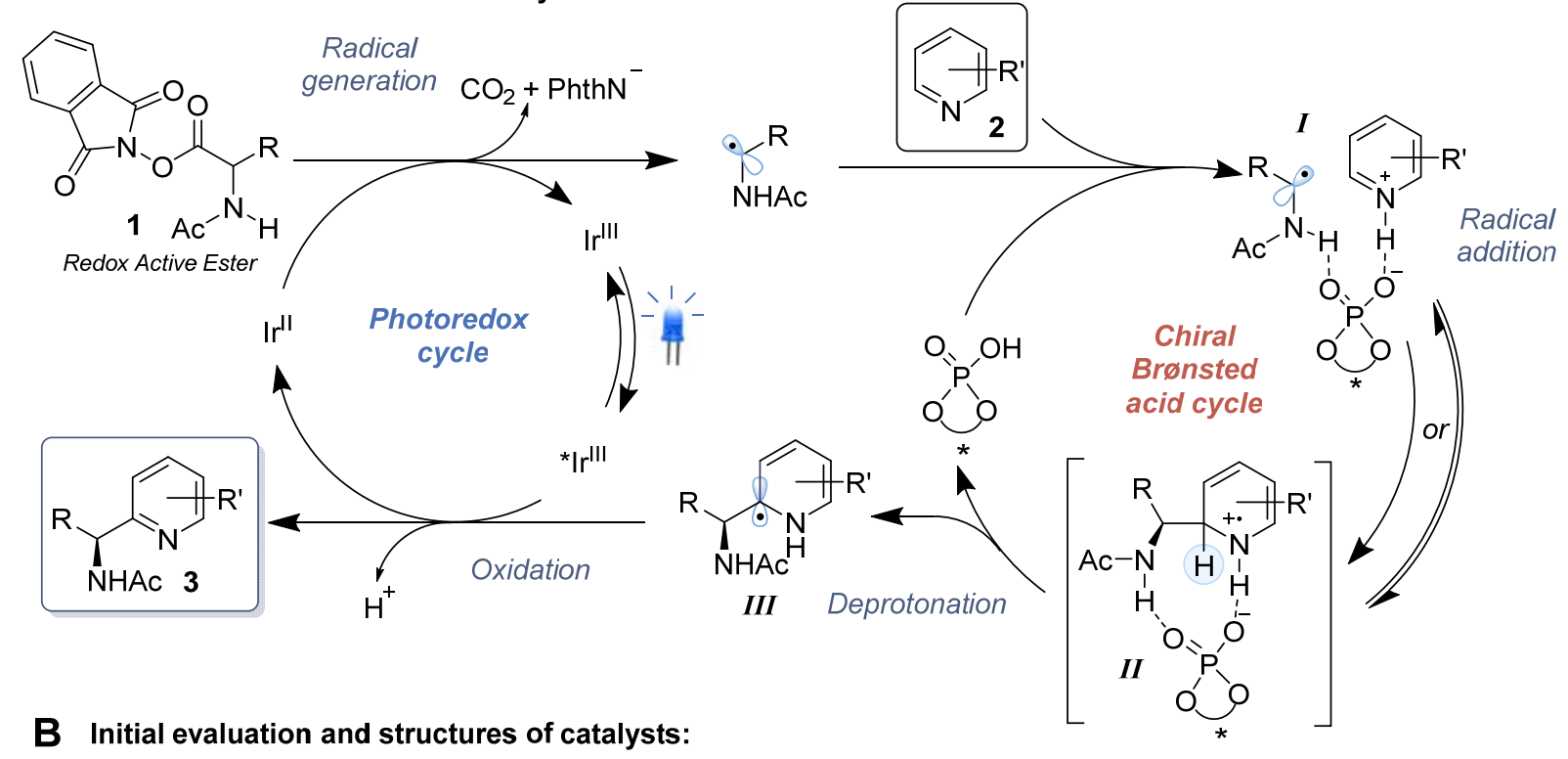

$2 \mathrm{~mol} \%\left[\operatorname{lr}\left(\mathrm{dF}\left(\mathrm{CF}_{3}\right) \text { ppy }\right)_{2}(\mathrm{dtbpy})\right] \mathrm{PF}_{6}$
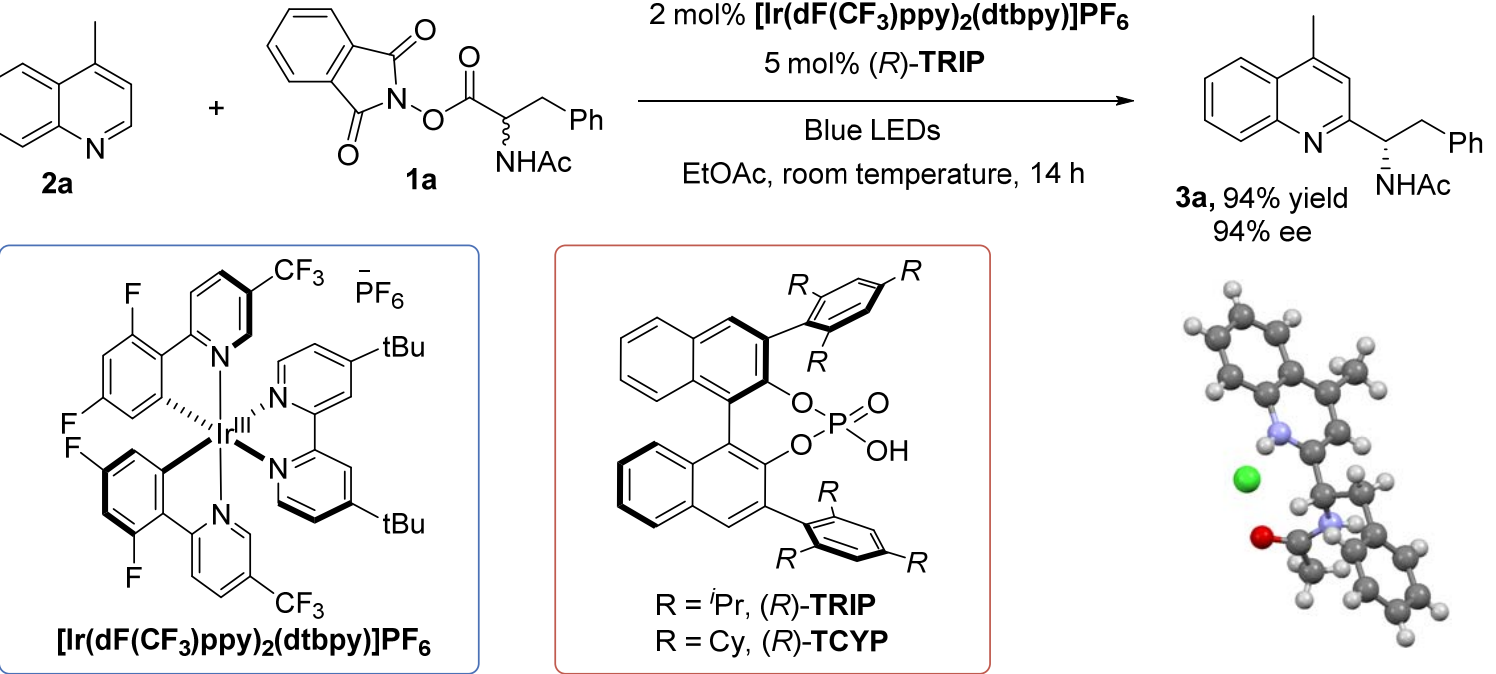

94\% ee

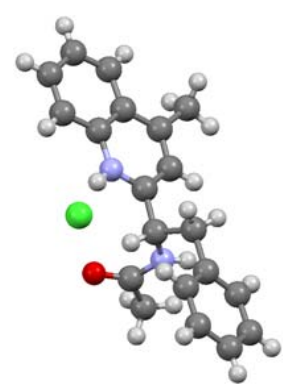

Fig. 2. Reaction development. (A) Hypothesis for enabling enantioselective Minisci-type addition. (B) Initial reaction evaluation on 4-methylquinoline, structures of the catalysts employed in this study and X-ray structure of 3a.

We elected to use redox-active esters (RAEs) derived from amino acids as precursors to $\mathrm{N}$ acyl $\alpha$-amino alkyl radicals (Fig 2A, radical generation), the latter being prochiral, nucleophilic radicals which possess hydrogen bond donor functionality. It is well established that facile alkyl radical generation occurs from RAEs on acceptance of a single electron (38-40) and there are a number of recent examples of photoredox catalysts being used as electron donors for this purpose, including in conjunction with RAEs derived from $N$-acyl amino acids $(20,41,42)$ and for Minisci-type additions (20). In line with these previous studies, we envisaged a reductive photoredox cycle, in which fragmentation of the RAE is induced via electron-transfer from an Ir(II) species generated off-cycle (for fluorescence quenching studies, see Figures S1 to S4 and 
accompanying text). After regeneration of $\operatorname{Ir}(\mathrm{III})$ and subsequent excitation, the catalyst should function as the single electron oxidant required to rearomatize the heteroarene (Fig 2A, III to 3).

To assess whether asymmetric induction could be achieved, we selected 4-methylquinoline as a test substrate. Irradiation from blue LEDs in the presence of $2 \mathrm{~mol} \%$ of photocatalyst

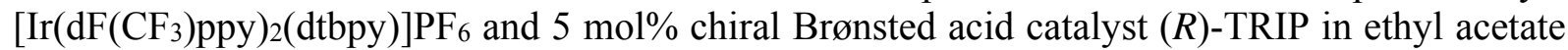
enabled efficient coupling of 4-methylquinoline (2a) and the RAE derived from $N$-acetyl-Lphenylalanine (1a) to give 3a in 94\% yield after 14h (Fig. 2B). The enantiomeric excess of the product was determined to be $94 \%$ and the absolute stereochemistry of the hydrochloride salt was determined by x-ray crystallography. Analysis of the RAE employed in this reaction showed that it was racemic, indicating that the stereocenter had epimerized during either synthesis or purification. We evaluated a selection of $\mathrm{N}$-protecting groups on the amino acid portion and acetyl was found to be optimal in terms of both yield and enantioselectivity (Table S1). A range of solvents were compatible, with dioxane found to give highest enantioselectivities, and the photocatalyst loading could be reduced to $1 \mathrm{~mol} \%$ (Tables S2 \& S3). Numerous $N$-acetyl amino acids are commercially available, as are both catalysts in this process, factors that contribute to the practicality of this procedure. Control experiments without the photocatalyst, Brønsted acid catalyst or visible light irradiation all gave no conversion to product (Table S4).

Encouraged by this result, we evaluated a range of amino acid-derived RAEs (Fig. 3A) and found the scope to be broad. Non-aromatic amino acids reacted with comparably high enantioselectivity; for example, the redox-active ester derived from $\mathrm{N}$-Ac-Alanine gave $94 \%$ ee (3b). Branching in the alkyl chain was well tolerated, as observed in the RAE derived from $\mathrm{N}$ Ac-Valine (3c, 97\% ee). The catalyst overrode influence from an adjacent stereocenter, as demonstrated in the application of each enantiomer of TRIP to the RAE derived from $\mathrm{N}$-AcIsoleucine (3d and 3e, both $>20: 1 \mathrm{dr}$ ). Remote arenes, esters and protected amines were well tolerated (3f, $\mathbf{3 g}$ and $\mathbf{3 h}$ ), as was the sulfide of methionine (3i). The carbon-iodine bond of iodotyrosine $(\mathbf{3} \mathbf{j})$ was untouched under the mild conditions, as was the electron-rich indole heterocycle in tryptophan (3k). O-Ac-Tyrosine was also appended effectively (31).

Although most of the RAEs used in Fig 3 were derived from enantiopure amino acids, almost all exhibited negligible optical rotation which we attribute to ready epimerization of the RAEs during synthesis or purification. The main exception to this was the RAE derived from valine, which appeared to be configurationally stable: the RAEs derived from L- and D- valine exhibited equal but opposite values of specific rotation and chiral HPLC analysis corroborated this observation. Control experiments with several substrates using $(R)$-TRIP showed that both RAEs gave the same enantiomer of product with the same enantiomeric excess, demonstrating that the stereochemistry of the RAE does not influence the stereochemical outcome (Tables S5 \& S6).

We next evaluated the scope of compatible quinolines (Fig. 3B). For unsubstituted quinoline, we anticipated that mixtures of regioisomers may arise as a result of competitive $\mathrm{C} 2$ - and $\mathrm{C} 4$ addition. However, we were pleased to discover that under our conditions very high $(>20: 1)$ regioselectivity for the $\mathrm{C} 2$ position was obtained, together with excellent enantioselectivity (3m, 96\% ee). Methoxy (3n), chloride (3o) and fluoride (3p) substituents were tolerated on the benzo portion and in all cases excellent regioselectivity was observed for addition at the $\mathrm{C} 2$ position. Regioselectivity in Minisci-type additions is known to be dependent on a range of factors, including solvent, acid and the nature of the radical $(37,43)$. In our case, use of a polar solvent ( $N, N$-dimethylacetamide) resulted in a switch to moderate $\mathrm{C} 4$ selectivity, in line with previous studies (20,37). Interestingly, use of acetyl as the $N$-protecting group on the RAE and TRIP as 
catalyst were both found to be crucial to achieving the very high observed regioselectivity for $\mathrm{C} 2$ addition; variation of either resulted in only moderate C2 selectivity (Tables S9 and S10). Substitution was well tolerated on the pyridine portion of the quinoline and can encompass 4phenyl (3q), 3-methyl (3r) and a fused benzene ring (3s). An aryloxy group could be included in the 4-position, which is particularly relevant in agrochemicals as diaryloxy compounds encompass a privileged family of herbicides (3t) (44). In contrast to the excellent enantioselectivity obtained with quinoline, isoquinoline gave significantly lower enantioselectivity (Scheme S1).

The scope was then explored on pyridines (Fig. 3C). These proved to be very amenable to our method and we observed the following: first, an electron-withdrawing substituent is typically necessary to enable productive radical addition and second, the highest enantioselectivities could be obtained by using the bulkier TCYP phosphoric acid (Fig. 2B, Tables S7 \& S8) (45). No addition at the $\mathrm{C} 4$ position was observed in any case. The substrate could feature ester $(\mathbf{3 u}, \mathbf{3 v}$, $\mathbf{3 x}$ ), ketone (3w), nitrile (3y) and trifluoromethyl (3z) groups at the 3- or 4- positions, together with other substituents around the ring, including methyls $(\mathbf{3 u}, \mathbf{3 x}, \mathbf{3 y})$, an unprotected amine (3za) and a bromide (3zb).

Minisci-type additions are one of the most valuable tools for late-stage functionalization of pharmaceuticals and we showed that our enantioselective addition works very effectively on two examples (Fig. 3D) (46). Metyrapone, an inhibitor of cortisol biosynthesis, was functionalized in a chemo-, regio- and enantioselective manner with excellent enantiomeric excess (3zc). Etofibrate, a combination of Clofibrate and niacin (3zd) also underwent highly selective functionalization at the pyridine ring. 


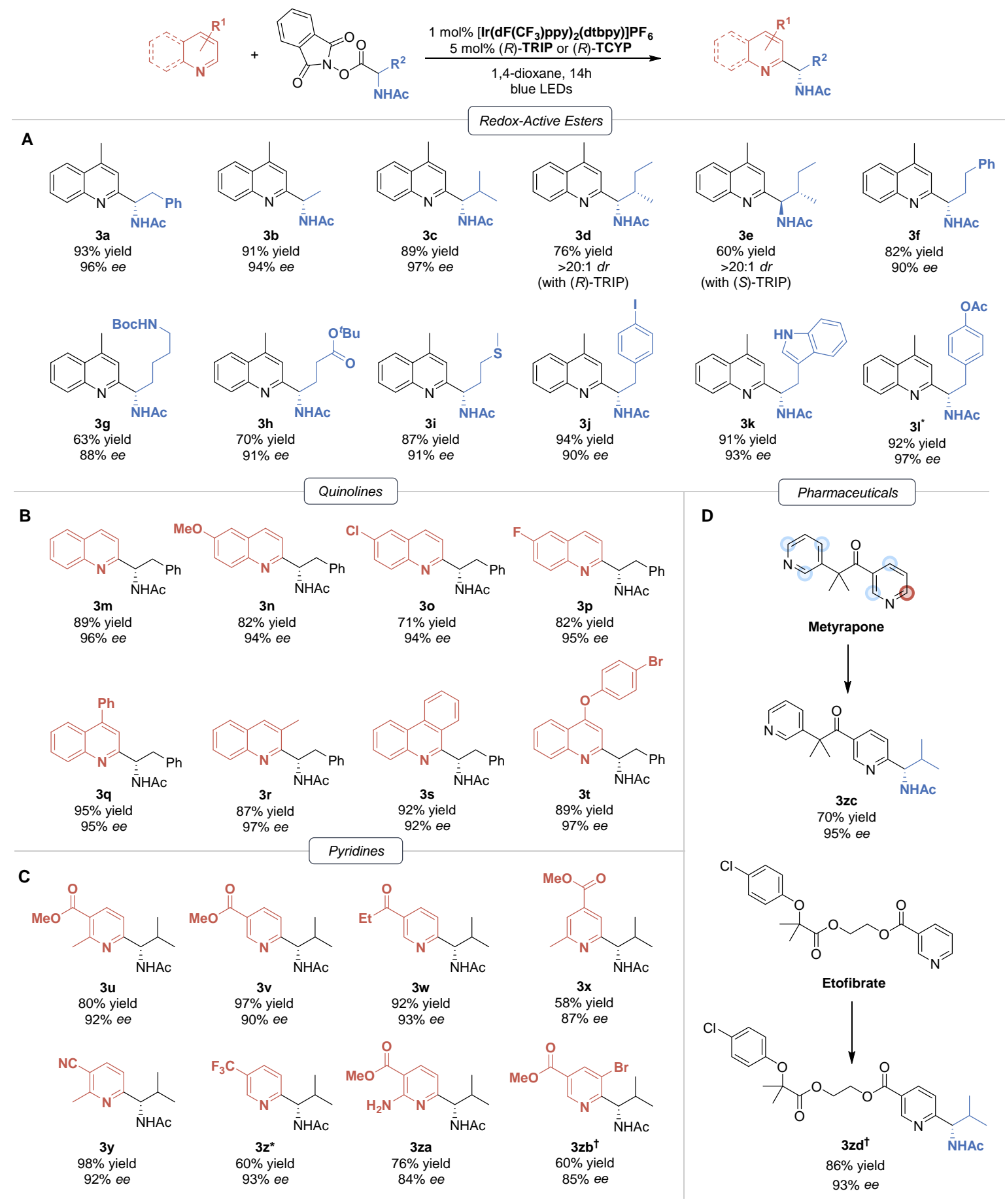

Fig. 3. Exploration of substrate scope. All yields are isolated yields, enantiomeric excesses determined by HPLC. Absolute stereochemistry of products assigned in analogy with 3a. (A) Evaluation of scope of redox active ester component. ${ }^{*} O$-Acetyl group was cleaved on purification. ${ }^{\dagger} \mathrm{mol} \%$ photocatalyst and $10 \mathrm{~mol} \%$ chiral Brønsted acid used. (B) Scope of 
quinoline component. (C) Scope of compatible pyridines. (D) Application to enantioselective late-stage functionalization of pharmaceuticals.

In order to provide some mechanistic insight, we performed some preliminary experiments. First, the redox-active ester derived from L-proline, which does not bear a hydrogen bond donor, delivered product 3ze in good yield but negligible enantioselectivity (Fig. 4A). This result supports the crucial role of attractive non-covalent interactions between the phosphate and either the radical nucleophile or a reaction intermediate such as II (Fig 2A). Second, we carried out a competition experiment between quinoline and quinoline- $d_{7}$ which demonstrated a primary kinetic isotope effect of 3.6. This outcome suggests that the second mechanistic scenario is most likely in operation: a potentially complex situation wherein radical addition may be reversible and the relative energies of diastereomeric intermediates $\boldsymbol{I I}$ and the rates of their deprotonation could both contribute to the excellent observed enantioselectivities (Fig. 4B) (37). Finally, we observed a non-linear relationship between the enantiopurity of catalyst and product, which suggests that two molecules of chiral phosphate may be involved in the deprotonation step (Figure S5).

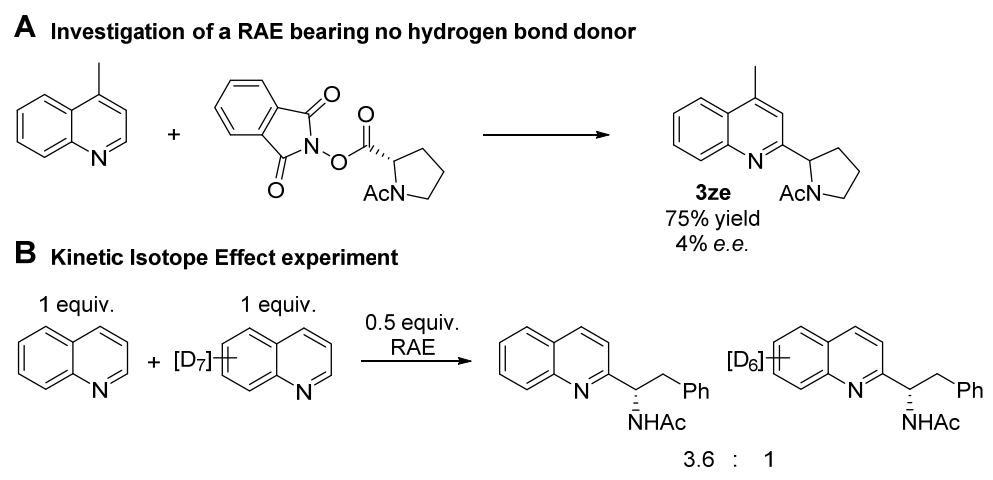

Fig. 4. Preliminary experiments to probe mechanism. (A) Outcome of reaction of a RAE that bears no hydrogen bond donors. (B) Kinetic isotope effect experiment.

Given the extraordinary pace of advancement of the field of photoredox catalysis and the range of molecules that are susceptible to Brønsted acid activation, we envisage that our successful merger of these strategies to address the challenge of enantioselective Minisci chemistry will have broad impact in both areas.

Acknowledgments: We are grateful to Professors Steven V. Ley and Matthew J. Gaunt for support and useful discussions. Thanks also to Dr. Eric P. Talbot (GSK), Jonathan Taylor (GSK) and Dr. David Blakemore (Pfizer) for useful discussion. We thank the EPSRC UK National Mass Spectrometry Facility at Swansea University and Dr. Andrew Bond for X-ray crystallography. Funding: We are grateful to the EPSRC and GlaxoSmithKline for a CASE studentship (R.S.J.P.), the EPSRC and Pfizer for a CASE studentship (H.J.D.), the EPSRC (EP/N005422/1) and the Royal Society for a University Research Fellowship (R.J.P.). Author 
contributions: R.S.J.P developed the reactions and performed and analyzed experiments. H.J.D. performed and analyzed experiments. R.J.P. conceived and supervised the project and wrote the manuscript, with input from all authors. Competing interests: None to declare. Data and materials availability: Supplementary materials contain additional data. All data needed to evaluate the conclusions in the paper are present in the paper or the supplementary materials. Crystallographic data are available free of charge from the Cambridge Crystallographic Data Centre under reference number CDC-1588695.

\section{Supplementary Materials:}

Materials and Methods

Figures S1 to S5

Tables S1 to S10

Schemes $\mathrm{S} 1$ to $\mathrm{S} 3$

References (47-58)

HPLC Traces

NMR Spectra

\section{References}

1. A. F. Pozharskii, A. T. Soldatenkov, A. R. Katritzky, in Heterocycles in Life and Society. (John Wiley \& Sons, Ltd, 2011).

2. E. Vitaku, D. T. Smith, J. T. Njardarson, J. Med. Chem. 57, 10257-10274 (2014).

3. F. Minisci, E. Vismara, F. Fontana, Heterocycles 28, 489-519 (1989).

4. F. Minisci, F. Fontana, E. Vismara, J. Heterocyclic Chem. 27, 79-96 (1990).

5. M. A. J. Duncton, Med. Chem. Commun. 2, 1135-1161 (2011).

6. J. Tauber, D. Imbri, T. Opatz, Molecules 19, 16190 (2014).

7. I. B. Seiple, S. Su, R. A. Rodriguez, R. Gianatassio, Y. Fujiwara, A. L. Sobel, P. S. Baran, J. Am. Chem. Soc. 132, 13194-13196 (2010). 
8. Y. Ji, T. Brueckl, R. D. Baxter, Y. Fujiwara, I. B. Seiple, S. Su, D. G. Blackmond, P. S. Baran, Proc. Natl. Acad. Sci. USA 108, 14411-14415 (2011).

9. G. A. Molander, V. Colombel, V. A. Braz, Org. Lett. 13, 1852-1855 (2011).

10. Y. Fujiwara, J. A. Dixon, F. O/'Hara, E. D. Funder, D. D. Dixon, R. A. Rodriguez, R. D. Baxter, B. Herle, N. Sach, M. R. Collins, Y. Ishihara, P. S. Baran, Nature 492, 95-99 (2012).

11. J. K. Matsui, D. N. Primer, G. A. Molander, Chem. Sci. 8, 3512-3522 (2017).

12. C. K. Prier, D. A. Rankic, D. W. C. MacMillan, Chem. Rev. 113, 5322-5363 (2013).

13. D. M. Schultz, T. P. Yoon, Science 343, 1239176 (2014).

14. $\quad$ N. A. Romero, D. A. Nicewicz, Chem. Rev. 116, 10075-10166 (2016).

15. J. Jin, D. W. C. MacMillan, Nature 525, 87-90 (2015).

16. C. A. Huff, R. D. Cohen, K. D. Dykstra, E. Streckfuss, D. A. DiRocco, S. W. Krska, J. Org. Chem. 81, 6980-6987 (2016).

17. J. Jin, D. W. C. MacMillan, Angew. Chem. Int. Ed. 54, 1565-1569 (2015).

18. G.-X. Li, C. A. Morales-Rivera, Y. Wang, F. Gao, G. He, P. Liu, G. Chen, Chem. Sci. 7, 6407-6412 (2016).

19. R. A. Garza-Sanchez, A. Tlahuext-Aca, G. Tavakoli, F. Glorius, ACS Catal. 7, 40574061 (2017).

20. W.-M. Cheng, R. Shang, Y. Fu, ACS Catal. 7, 907-911 (2017).

21. W.-M. Cheng, R. Shang, M.-C. Fu, Y. Fu, Chem. Eur J. 23, 2537-2541 (2017).

22. F. Lovering, J. Bikker, C. Humblet, J. Med. Chem. 52, 6752-6756 (2009).

23. J. Maury, W. Zawodny, J. Clayden, Org. Lett. 19, 472-475 (2017).

24. J. Llaveria, D. Leonori, V. K. Aggarwal, J. Am. Chem. Soc. 137, 10958-10961 (2015).

25. P. D. O'Shea, D. Gauvreau, F. Gosselin, G. Hughes, C. Nadeau, A. Roy, C. S. Shultz, J. Org. Chem. 74, 4547-4553 (2009).

26. Z. Zuo, H. Cong, W. Li, J. Choi, G. C. Fu, D. W. C. MacMillan, J. Am. Chem. Soc. 138, 1832-1835 (2016).

27. M. Silvi, P. Melchiorre, Nature 554, 41-49 (2018).

28. A. Bauer, F. Westkamper, S. Grimme, T. Bach, Nature 436, 1139-1140 (2005).

29. L. J. Rono, H. G. Yayla, D. Y. Wang, M. F. Armstrong, R. R. Knowles, J. Am. Chem. Soc. 135, 17735-17738 (2013).

30. G. Bergonzini, C. S. Schindler, C.-J. Wallentin, E. N. Jacobsen, C. R. J. Stephenson, Chem. Sci. 5, 112-116 (2014).

31. D. Uraguchi, N. Kinoshita, T. Kizu, T. Ooi, J. Am. Chem. Soc. 137, 13768-13771 (2015).

32. J.-S. Lin, X.-Y. Dong, T.-T. Li, N.-C. Jiang, B. Tan, X.-Y. Liu, J. Am. Chem. Soc. 138, 9357-9360 (2016).

33. L. Lin, X. Bai, X. Ye, X. Zhao, C.-H. Tan, Z. Jiang, Angew. Chem. Int. Ed. 56, 1384213846 (2017).

34. M. Rueping, D. Parmar, E. Sugiono, Asymmetric Bronsted Acid Catalysis. (Wiley, 2015).

35. C. A. Correia, L. Yang, C.-J. Li, Org. Lett. 13, 4581-4583 (2011).

36. J. L. Biaco, S. L. Jones, T. J. Barker, Heterocycles 92, 1687-1697 (2016).

37. F. Minisci, E. Vismara, F. Fontana, G. Morini, M. Serravalle, C. Giordano, J. Org. Chem. 52, 730-736 (1987).

38. K. Okada, K. Okamoto, N. Morita, K. Okubo, M. Oda, J. Am. Chem. Soc. 113, 9401 9402 (1991). 
39. G. Pratsch, G. L. Lackner, L. E. Overman, J. Org. Chem. 80, 6025-6036 (2015).

40. T. Qin, J. Cornella, C. Li, L. R. Malins, J. T. Edwards, S. Kawamura, B. D. Maxwell, M.

D. Eastgate, P. S. Baran, Science 352, 801-805 (2016).

41. J. Schwarz, B. Konig, Green Chem. 18, 4743-4749 (2016).

42. Y. Jin, M. Jiang, H. Wang, H. Fu, Sci. Rep. 6, 20068 (2016).

43. F. O'Hara, D. G. Blackmond, P. S. Baran, J. Am. Chem. Soc. 135, 12122-12134 (2013).

44. E. Kennepohl, I. C. Munro, J. S. Bus, in Hayes' Handbook of Pesticide Toxicology (Third Edition). (Academic Press, New York, 2010), pp. 1829-1847.

45. V. Rauniyar, Z. J. Wang, H. E. Burks, F. D. Toste, J. Am. Chem. Soc. 133, 8486-8489 (2011).

46. T. Cernak, K. D. Dykstra, S. Tyagarajan, P. Vachal, S. W. Krska, Chem. Soc. Rev. 45, 546-576 (2016).

47. H. J. Cahnmann, Anal. Chem. 29, 1307-1311 (1957).

48. M. Klussmann, L. Ratjen, S. Hoffmann, V. Wakchaure, R. Goddard, B. List, Synlett 2010, 2189-2192 (2010).

49. A. Singh, K. Teegardin, M. Kelly, K. S. Prasad, S. Krishnan, J. D. Weaver, J. Organomet. Chem 776, 51-59 (2015).

50. I. Regla, H. Luna, H. I. Pérez, P. Demare, I. Bustos-Jaimes, V. Zaldívar, M. L. Calcagno, Tetrahedron: Asymmetry 15, 1285-1288 (2004).

51. R. C. Wende, A. Seitz, D. Niedek, S. M. M. Schuler, C. Hofmann, J. Becker, P. R. Schreiner, Angew. Chem. Int. Ed. 55, 2719-2723 (2016).

52. G. Naturale, M. Lamblin, C. Commandeur, F.-X. Felpin, J. Dessolin, Eur. J. Org. Chem. 2012, 5774-5788 (2012).

53. M. Kalek, G. C. Fu, J. Am. Chem. Soc. 137, 9438-9442 (2015).

54. J. Yuan, J.-T. Yu, Y. Jiang, J. Cheng, Org. Biomol. Chem. 15, 1334-1337 (2017).

55. K. Okada, K. Okamoto, M. Oda, J. Am. Chem. Soc. 110, 8736-8738 (1988).

56. M. S. Lowry, J. I. Goldsmith, J. D. Slinker, R. Rohl, R. A. Pascal, G. G. Malliaras, S. Bernhard, Chem. Mater. 17, 5712-5719 (2005).

57. W. Tang, S. Johnston, J. A. Iggo, N. G. Berry, M. Phelan, L. Lian, J. Bacsa, J. Xiao, Angew. Chem. Int. Ed. 52, 1668-1672 (2013).

58. A. Tlahuext-Aca, R. A. Garza-Sanchez, F. Glorius, Angew. Chem. Int. Ed. 56, 3708-3711 (2017). 